\title{
Desalination Performance of Modified Multiwall Carbon Nanotubes
}

\author{
Received in revised form 2 March 2005, accepted 3 March 2005 \\ Dengsong Zhang ${ }^{1,2}$, Liyi Shi ${ }^{1,2^{*}}$, Jianhui Fang ${ }^{1,2}$ \& Kai Dai ${ }^{1}$ \\ ${ }^{1}$ School of Material Science and Engineering, Shanghai University, Shanghai 200444, China \\ ${ }^{2}$ School of Science, Shanghai University, Shanghai, 200444, China
}

\begin{abstract}
Modified multiwall carbon nanotubes (MWCNTs) were used as the electrodes of flow-through capacitor for the desalination, which were cost-cutting, energy-saving, and environment-friendly and could be expected to be an alternative technology for reverse osmosis membrane for sea water desalination. Investigated by transmission electron microscope (TEM) and nitrogen adsorption/desorption for modified MWCNTs, it was confirmed that after modification treatment by immersion in diluted $\mathrm{HNO}_{3}$ solution with ultrasonic and then milling by ball at a high velocity, the metal catalyst particles at the tip of MWCNTs disappeared, the MWCNT length became short, the cap at the tip of nanotube was opened, the internal surface area could be effectively used, leading to the increase of the specific surface area and pore volume, and thus MWCNTs modified by that method had the best desalination performance. This modification technique is simple and the efficiency is high.
\end{abstract}

Keywords : Carbon nanotube, modification, desalination, membrane

\subsection{INTRODUCTION}

Water resources crisis is one of the biggest resources crises in the century, in the world. Seawater desalination is an important way in solving this crisis. There are various techniques for seawater desalination such as seawater reverse osmosis, multi-stage flash, multiple effect distillation, vapor compression distillation, and electrodialysis [1-5]. In recent years, desalination with reverse osmosis membrane has been developed as one of the most important techniques to reduce the cost of seawater desalination:

In 1990's, Andelman mentioned a new seawater desalination method using flow-through capacitor by extending the concept of the double layer capacitor seen from Figure 1, which made use of an electricity layer principle, regarding activated carbon as the electrodes [6]. However, activated carbon with high resistivity had many micropores which hydrated ion could not entered into, which had a bad effect on the desalination. This resulted in early versions using activated carbon as electrode not saving much energy nor work well at high concentrations [7-9]. The high surface area materials such as graphite, acetylene black, noble mentals, conductive ceramics, and conductive polymers were

\footnotetext{
* Correspondence to: Liyi Shi (fax:+86-21-66135066, e-mail: sly0726@163.com)
} 


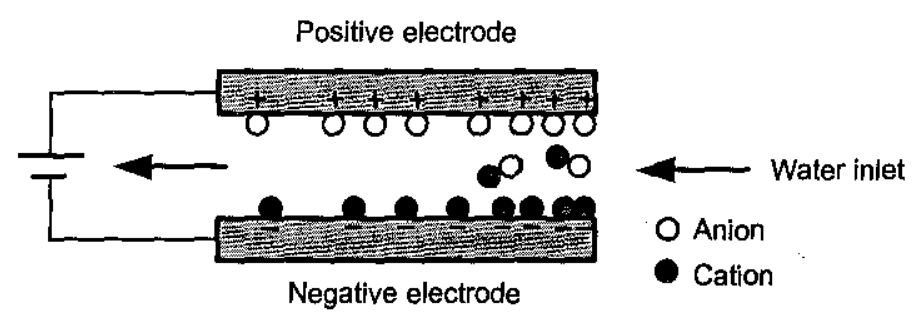

Figure 1 Simple flow-through capacitor

also mentioned in flow through capacitor but the desalination performances were not satisfied [10, 11]. Carbon nanotubes have a narrow distribution size, highly accessible surface areas, low resistivity, and high stability. Considerable theoretical and experimental investigations on their novel structure and applications had been carried out [12-18]. Shi, L. et al. [19] had patented using carbon nanotubes in the fabrication of flow-through capacitor, which was applied to desalt seawater.

This desalination technique which was cost-cutting, energy-saving, and environment-friendly as compared to other above-mentioned technologies, could be expected to be an alternative technology for reverse osmosis membrane for sea water desalination. In this paper, the influence of MWCNTs with different modification methods on the desalination performance was investigated.

\subsection{EXPERIMENTAL}

\subsection{Raw Materials}

MWCNTs synthesized by the catalytic decomposition of methane were supplied by Shenzhen Nanotech Port Co., Ltd. Activated carbon was supplied by Chaoyang Activated Carbon Co., Ltd. Phenolic resin and urotropine were supplied by Shanghai SANAIFU Co., Ltd.

\subsection{Modification Of MWCNTs}

Raw MWCNTs were marked as sample A. The raw MWCNTs were immersed in $20 \% \mathrm{HNO}_{3}$ solution with ultrasonic for 2 hours, then washed several times with distilled water on a sintered glass filter until the washings showed no acidity. Finally, it was dried in an oven at $100^{\circ} \mathrm{C}$ for 24 hours. The product was marked as sample B. Circumfluence in concentrated $\mathrm{HNO}_{3}$ and $\mathrm{H}_{2} \mathrm{SO}_{4}$ solution was employed to MWCNTs for 0.5 hours, and then MWCNTs were washed several times with distilled water on a sintered glass filter until the washings showed no acidity. Then it was dried in an oven at $100^{\circ} \mathrm{C}$ for 24 hours and sample $\mathrm{C}$ was obtained. As oxidization in air atmosphere could open the tip of carbon nanotubes and increase their surface area [20], sample B was oxidized in air atmosphere at $600^{\circ} \mathrm{C}$ for 0.5 hours, and the product was marked as sample D. After being milled by ball at a high velocity as $1200 \mathrm{r} / \mathrm{min}$ for 2 hours, sample B was marked as sample $\mathrm{E}$.

\subsection{Preparation of MWCNT Electrodes}

The mixture of modified MWCNTs and binder powders in a weight ratio of 80:20 was molded under $25 \mathrm{MPa}$ pressure at $150^{\circ} \mathrm{C}$ for 15 minutes, where the binders were made up of phenolic resin (90\%) 
and urotropine $(10 \%)$, and then the tablet was carbonized at $850^{\circ} \mathrm{C}$ for 2 hours under nitrogen atmosphere. Finally, the electrodes were obtained after natural cooling. Activated carbon electrode was fabricated by using the same method. All electrodes were $115 \mathrm{~mm}$ in length, $75 \mathrm{~mm}$ in width, and about $1 \mathrm{~mm}$ in thickness.

\subsection{Apparatus of Flow-through Capacitor}

The apparatus of flow-through capacitor shown in Figure 2 was employed. Graphite foil was used as an inert current collector on the backside of the MWCNT electrode, and the electrodes were separated by the separator into anode/cathode pairs. $2000 \mathrm{mg} / \mathrm{L}$ saltwater solution was passed through the capacitor at a flow rate of $10 \mathrm{ml} / \mathrm{min}$. NaCl was removed by applying a direct voltage of $1 \mathrm{~V}$ between the collectors. In order to examine the desalination effect of the apparatus with precision, the removal process was started after the concentration of the saltwater solution exiting the apparatus was constant. After a long time operation, the electrode was saturated with the ions and thus the outlet concentration increases. For regeneration of the MWCNT electrodes, a reverse voltage of $1 \mathrm{~V}$ was applied to both collectors to desorb and recover captured concentrated saltwater solution.

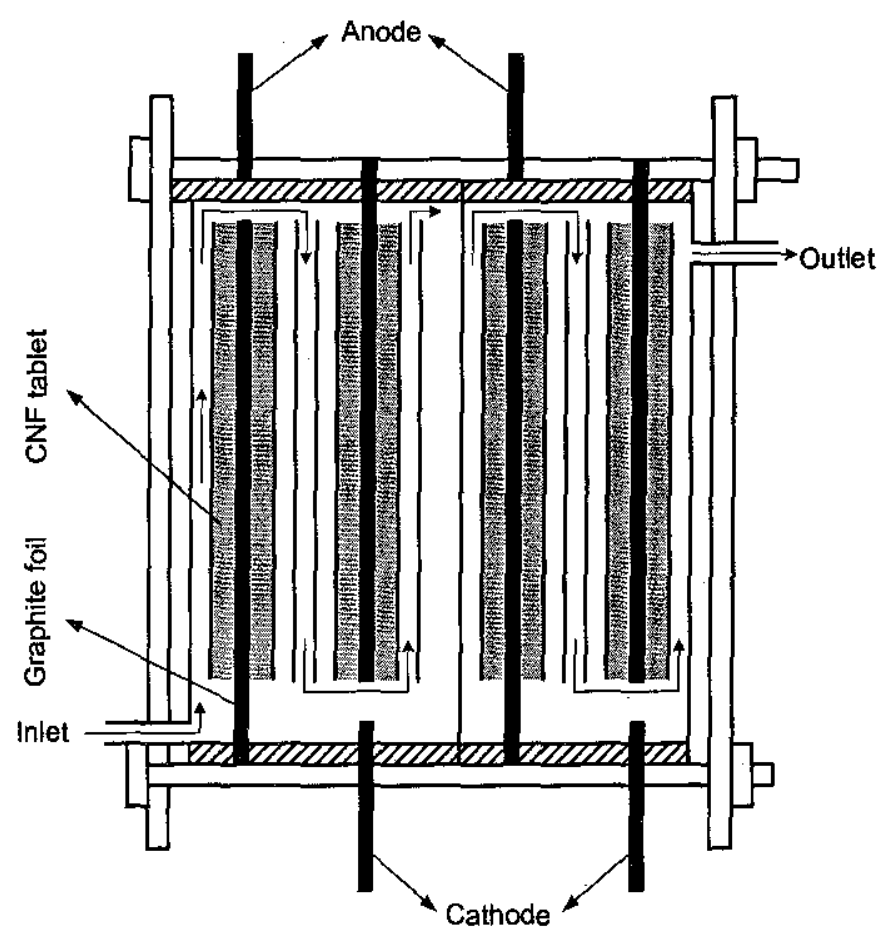

Figure 2 The sketch of desalinator

\subsection{Test and Measurement}

MWCNT powders were observed by TEM of JEOL JEM-2010, and powdered samples were dispersed in absolute ethanol by ultrasonication for $10 \mathrm{~min}$ in a KQ-250B ultrasonic bath. The surface texture of all samples such as surface area and pore volume, were characterized by nitrogen adsorption at 77 
K with Micromeritics ASAP 2010. The CyberScan CON 200 conductivity meter was employed to directly get the data of desalination.

\subsection{RESULTS AND DISCUSSION}

Different desalination curves of electrodes fabricated by modified MWCNTs and activated carbon are shown in Figure 3, where $C$ is the outlet concentration, $C_{0}$ is the inlet concentration, and $C / C_{0}$ is the relative concentration. The surface texture of $\mathrm{A} \sim \mathrm{E}$ samples is listed in Table 1 . It could be seen that the electrode made of modified MWCNTs with the highest surface area and pore volume had the

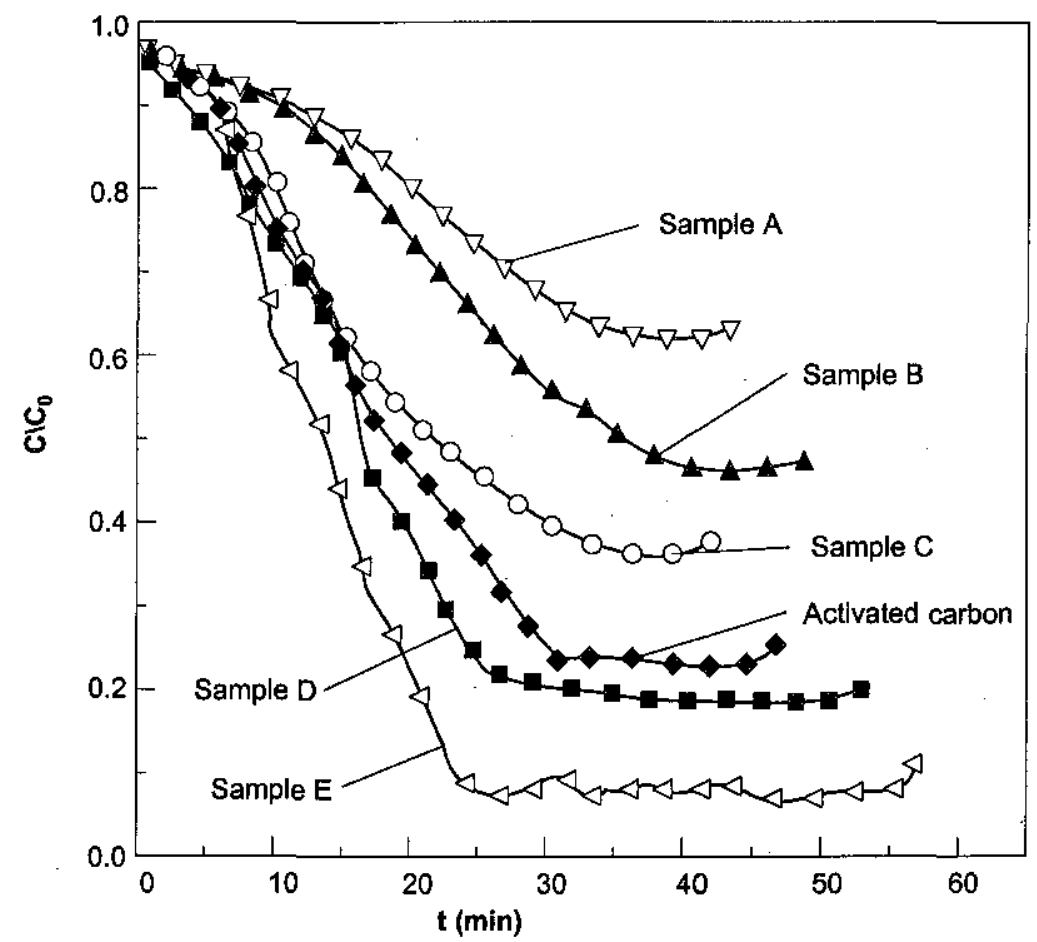

Figure 3 The desalination curves of different electrodes

Table 1 The surface texture of MWCNTs with different modification methods

\begin{tabular}{clcc}
\hline Sample & Modification methods & $\begin{array}{c}\text { Surface area } \\
\left(\mathbf{m}^{2} / \mathbf{g}\right)\end{array}$ & $\begin{array}{c}\text { Pore volume } \\
\left(\mathbf{c m}^{3} / \mathbf{g}\right)\end{array}$ \\
\hline A & Untreatment & 49.9 & 0.077 \\
B & Immersion in diluted $\mathrm{HNO}_{3}$ solution with ultrasonic & 55.5 & 0.103 \\
C & Circumfluence in concentrated $\mathrm{HNO}_{3}$ and $\mathrm{H}_{2} \mathrm{SO}_{4}$ solutions & 75.3 & 0.186 \\
D & Immersion in diluted $\mathrm{HNO}_{3}$ solution with ultrasonic and & 91.2 & 0.165 \\
& then oxidization in air atmosphere & & \multirow{2}{*}{0.400} \\
E & Immersion in diluted $\mathrm{HNO}_{3}$ solution with ultrasonic an & & \\
& then milling by ball at a high velocity & \\
\hline
\end{tabular}


best desalination performance. Compared with activated carbon electrode in Figure 3, sample $\mathrm{E}$ had a better desalination performance, which was the reason why carbon nanotubes were chosen as the alternative electrode materials of flow-through capacitor.

In this study, MWCNTs synthesized by the catalytic decomposition of methane contained catalyst particles and other impurities. Figure 4 shows the TEM image of MWCNTs, which shows that the diameter was about $40 \sim 60 \mathrm{~nm}$. Some metal catalyst particles were encapsulated at the tip of the nanotube and the tip was closed, and thus the inner surface area could not be used effectively. Therefore, modification was a critical step in the use of MWCTs as electrodes materials. TEM image of sample $\mathrm{E}$ in Figure 5 shows that the metal catalyst particles at the tip of MWCNTs disappeared, the length of nanotubes became short, and the cap at the tip of nanotube was opened. Diluted $\mathrm{HNO}_{3}$ solution could dissolve catalyst particles with ultrasonic and thus purified MWCNTs were obtained, but their surface area increased a little. Generally, MWCNTs possessed many defects, such as localized kinks or bends. During ball milling, frequent collision and the high velocity of the balls caused large impacts on the MWCNTs. If the collision energy was high enough, the cylinder structure of the nanotubes would cracked at the site impacted and the tube layer would ruptured completely. Thus nanotubes with open ends could be formed. Milled nanotubes were prone to break at the sites of

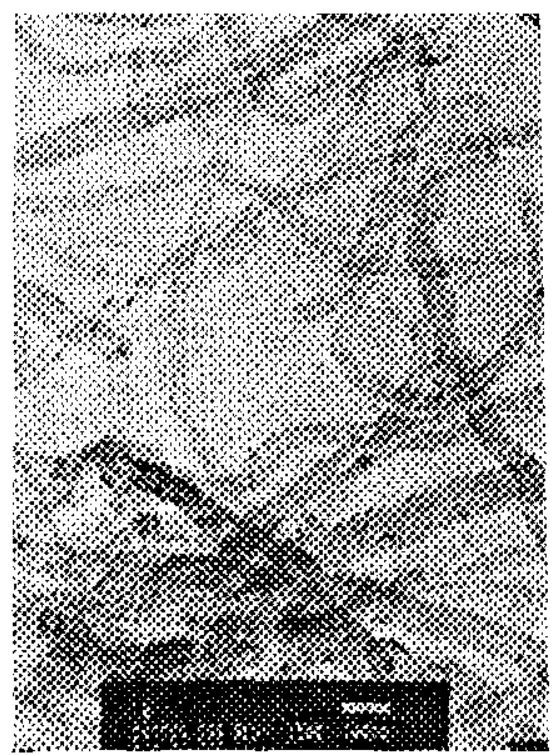

Figure 4 TEM image of sample A

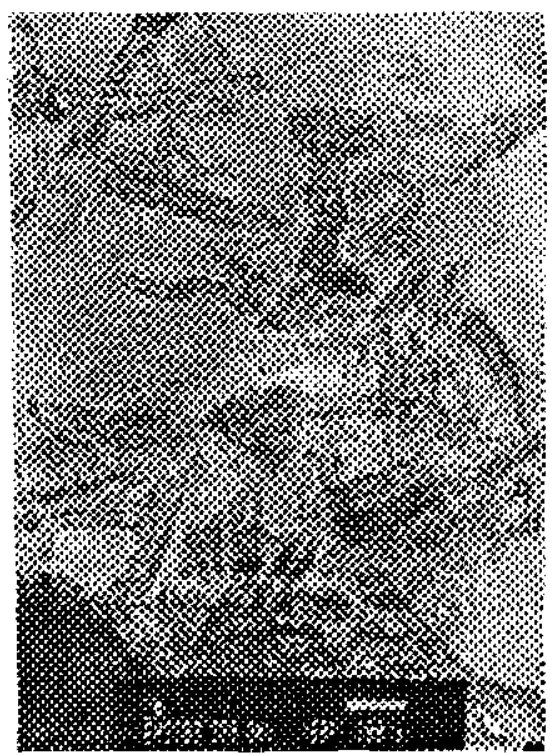

Figure 5 TEM image of sample E

structural defects, such as bends, thus causing the length of majority of milled nanotubes to become short [21]. Consequently, the internal surface area could be effectively used, leading to the increase of the specific surface area and pore volume. As other methods used in sample B, C, and D could not effectively cut the nanotubes, the internal surface area could not be used effectively, and thus their surface areas and pore volume were inferior to that of sample E. Compared with other modification in literatures [22-24], the modification technique used in sample $\mathrm{E}$ was simple and the efficiency was high. After this modification on MWCNTs, the mechanical properties of the final electrode still remained.

MWCNTs with different modification methods in Figure 6 present a type IV nitrogen adsorption isotherm with a hysteresis loop typical of a mesoporous material, where the desorption required 


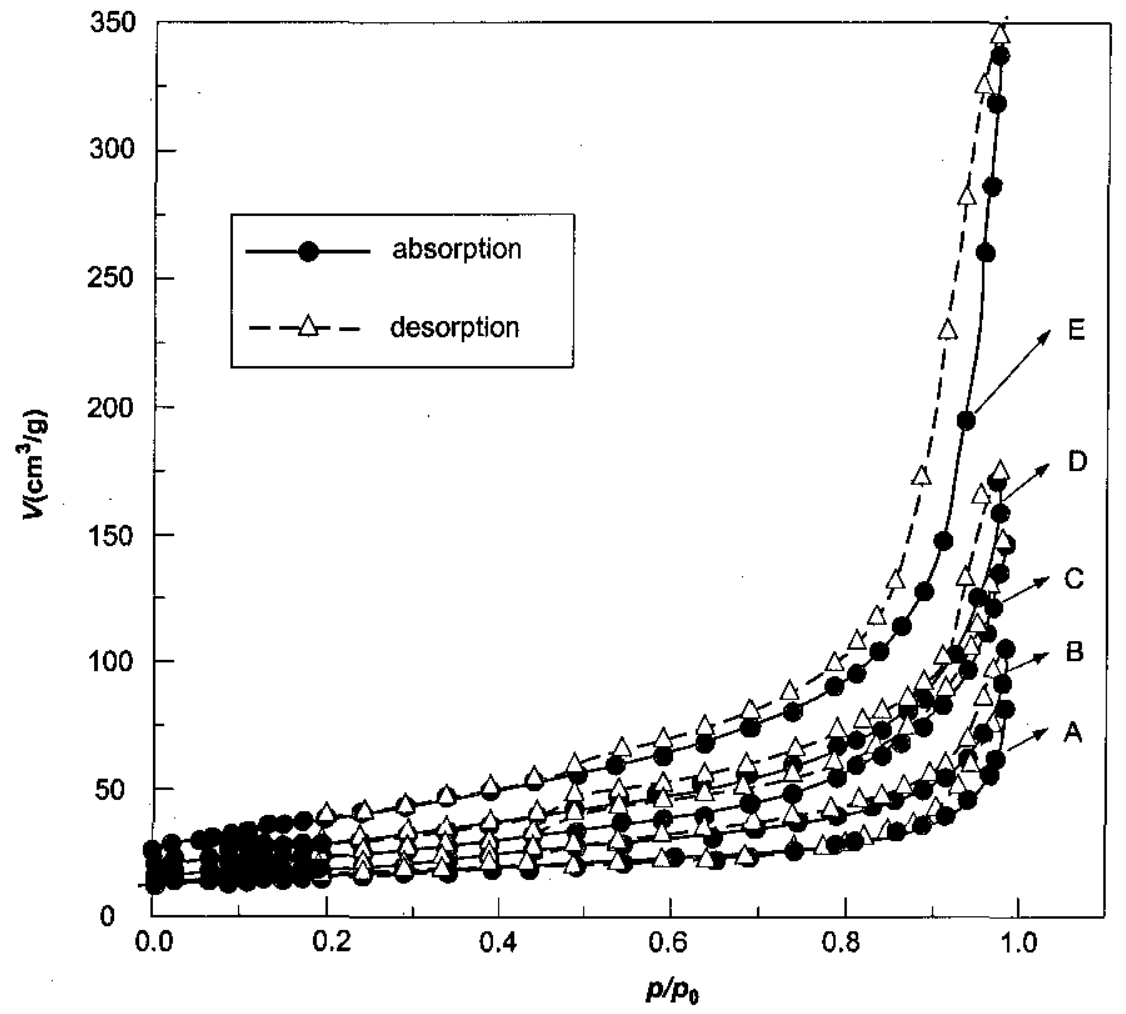

Figure 6 Nitrogen adsorption/desorption at $77 \mathrm{~K}$ for modified MWCNTs

definitively higher energy than adsorption [25]. The hysteresis was definitively more pronounced in the case of sample $\mathrm{E}$, which confirmed a wide distribution of pores. After the modification, the specific surface area of MWCNTs increased from 49.9 to $128.5 \mathrm{~m}^{2} / \mathrm{g}$ and the pore volume increased from 0.077 to $0.400 \mathrm{~m}^{3} / \mathrm{g}$, as shown in Table 1 , which verifies the importance of modification of MWCNTs.

Generally, the electrostatic capacity of double layer capacitor depended on the surface area of the electrode, as follows [26]:

$$
C=\int \varepsilon / 4 \pi \delta \cdot d S
$$

where $\varepsilon$ is the permittivity of solution, $\delta$ is the distance between the electrode and center of ion, and $S$ is the surface area of the electrode. Therefore, the desalination of the electrode increased with the surface area of the electrode. In general, the surface area of the electrodes increased with the specific surface areas of MWCNTs. As a result, the electrode made with sample $E$ with the highest surface area had the best desalination performance.

\subsection{CONCLUSIONS}

Using modified MWCNTs as the electrodes of flow-through capacitor, it was possible to efficiently remove $\mathrm{NaCl}$ from saltwater solution. This technique was cost-cutting, energy-saving, and 
environment-friendly and could be expected to be an alternative technology for reverse osmosis membrane for sea water desalination. The results showed that the modifications of MWCNTs had a great effect on the desalination performance. It was confirmed that after modification treatment by immersion in diluted $\mathrm{HNO}_{3}$ solution with ultrasonic and then milling by ball at a high velocity, the metal catalyst particles at the tip of MWCNTs disappeared, the MWCNT length became short, the cap at the tip of nanotube was opened, and the internal surface area could be effectively used, leading to the increase of the specific surface areas and pore volume. The amount of $\mathrm{NaCl}$ removal was generally dependent on the surface area and pore volume of MWCNTs.

\section{ACKNOWLEDGEMENTS}

The authors acknowledged the supports of the National High Technology Research and Development Program (863 Program) of China (2002AA302302) and Shanghai NanoScience \& Technology Special Project (0223nm001).

\section{REFERENCES}

[1] Reddy, B. et al. 1997. Design of Centralized Controllers for a MSF Desalination Plant. Desalination. 113(1): 27-38.

[2] Semiat, R., and Y. Galperin. 2001. Effect of Non-condensable Gases on Heat Transfer in the Tower MED Seawater Desalination Plant. Desalination. 140(1): 27-46.

[3] El-Sayed, and M. Yehia. 1999. Thermoeconomics of Some Options of Large Mechanical Vaporcompression Units. Desalination. 125(1-3): 251-257.

[4] Grundisch, A., and B. Schneider. 2001. Optimising Energy Consumption in SWRO Systems with Brine Concentrators. Desalination. 138(1-3): 223-229

[5] Demircioglu, M. et al. 2003. Demineralization by Electrodialysis (ED) - Separation Performance and Cost Comparison for Monovalent Salts. Desalination. 153(1-3): 329-333.

[6] Andelman, M. 1993. Flow-through Capacitor. US Patent 5192432.

[7] Andelman, M. 1996. Method of Separating Fluids with a Flow Through Capacitor. US Patent 5547561.

[8] Andelman, M. 2001. Energy and Weight Efficient Flow-through Capacitor System and Method. US Patent 6325907B1.

[9] Oda, H., and Y. Nakagawa. 2003. Removal of Ionic Substances from Dilute Solution Using Activated Carbon Electrodes. Carbon. 41: 1037-1047.

[10] Andelman, M. 1995. Flow-through Capacitor. US Patent 5415768.

[11] Noack, A. 2002. Apparatus for Purifying Water Comprises a Housing and Includes and Electrically Charges Electrodes Comprising a Porous Carbon Membrane on Porous Support. DE Patent 1003457.

[12] Odani, A. et al. 2003. Development and Testing of Nanomaterials for Rechargeable Lithium Batteries. Journal of Power Sources. 119-121: 517-521.

[13] Simonyan, V., and J. Johnson. 2003. Hydrogen Storage in Carbon Nanotubes and Graphitic Nanofibers. Journal of Alloys and Compounds. 330-332: 659-665.

[14] Nakayama, Y., and S. Akita. 2001. Field-emission Device with Carbon Nanotubes for a Flat Panel Display. Synthetic Metals. 117(1-3): 207-210.

[15] Cantalini, C. et al. 2004. Carbon Nanotubes as New Materials for Gas Sensing Applications. Journal of the European Ceramic Society. 24(6): 1405-1408. 
Dengsong Zhang, Liyi Shi, Jianhui Fang \& Kai Dai

[16] Chen, J. et al. 2002. Electrochemical Characterization of Carbon Nanotubes as Electrode in Electrochemical Double-layer Capacitors. Carbon. (40): 1193-1197.

[17] Frackowiak, E. et al. 2002. Nanotubular Materials as Electrodes for Supercapacitors. Fuel Processing Technology. 77-78: 213-219.

[18] Jiang, Q. et al. 2002. A Study of Activated Carbon Nanotubes as Electrochemical Super Capacitors Electrode Materials. Materials Letters. 57: 988-991.

[19] Shi, L. et al. 2003. A Method About Seawater Desalination Using Flow Through Capacitor with Carbon Nanotube Electrode. CN Patent 1463927A.

[20] Chunye, Z. et al. 2003. Review of Carbon Nanotubes Purification. Chemical Industry and Engineering Progress. 22(5): 482-485. (In Chinese).

[21] Fu, L. et al. 2003. Preparation of Short Carbon Nanotubes by Mechanical Ball Milling and Their Hydrogen Adsorption Behaviour. Carbon. 41: 2527-2532.

[22] Zhang, Y. et al. 2000. Structure Modification of Single-wall Carbon Nanotubes. Carbon. 38: 2055-2059.

[23] Colomer, J. et al. 1999. Different Purification Methods of Carbon Nanotubes Produced by Catalytic Synthesis. Synthetic Metals. 103: 2482-2483.

[24] Saito, T., K. Matsushige, and K. Tanaka. 2002. Chemical Treatment and Modification of Multiwalled Carbon Nanotubes. Physica B. 323: 280-283.

[25] Frackowiak, E. et al. 2002. Enhanced Capacitance of Carbon Nanotubes Through Chemical Activation. Chemical Physics Letters. 36: 35-41.

[26] Nishino, A. 1988. Carbon and Electronics - Electrical Double-layer Capacitor Using Activated Carbon Fiber. Tanso. 132: 57-71. 